\title{
ACTION OF AMINES AND GRIGNARD REAGENTS ON SOME NEW N- ARYLIDENEAMINOPHTHALIMIDES
}

\author{
H.T. Zaky \\ Chemistry Department. College for Women. Ain Shams Univeristy. Helipolis. Cairo.
}

Egypt.

\begin{abstract}
Some new $\mathrm{N}$-arylmethyleneaminophthalimides have been synthesized from the interaction of $\mathrm{N}$ - aminophthalimides(I) with a varity of carbonyl compounds such as aliphatic and aromatic aldehydes and ketones. The effect of $\mathrm{N}$-aminophthalimide on itaconic and citraconic anhydrides and the action of amines and Grignard reagent on some synthesized $\mathrm{N}$ arylmethyleneaminophthalimides have been also reported.
\end{abstract}

\section{Introduction}

$\mathrm{N}$-arylmethyleneaminophthalimides are reported to posses chemotheropeutic activity where it exhibit antibacterial [1]. antifungal [2]. adrenolytic [3] and schistosomicidal [4] proporties. As a part of a research program directed towards the synthesis of some new-Narylidenaminophthalimides of expected biological activity. we have previously described the chemistry of $\mathrm{N}$-aminophthalimide and some allied compounds [5-9].

\section{Results and discussions}

Continuing our research in thies area. it has been found that reaction of $\mathrm{N}$ methyleneaminophthalimides (I) with crotonaldehyde yielded the normal condensation product $\mathrm{N}$-crotonylmethyleneaminophthalimide (II) as a major product together with a minor amount of (III) which is thought to be intially formed and loose water to yield (II). This was confirmed by the fact that boiling of (III) in ethanol afforded (II) in a good yield. also. reaction of (I) with crotonaldehyde under prolonged reflux time (II) was the only product formed.

We also reported that compound (I) condensed with some aromatic aldehydes namely. quinoline-2-aldehyde. trimethoxybenzaldehyde. mesitylaldehyde and p-acetamedobenzaldehyde to give $\mathrm{N}$-substituted products $\left(\mathrm{IV}_{2-\mathrm{d}}\right)$. Also. when terephthaldehyde was allowed to react with two moles of (I). compound (V) was obtained (Scheme I).

On the other hand. when equimolar amounts of (I) and diacetyl was refluxed in ethanol. compound (VI) was produced which confirmed by 'HNMR (DMSO): 7.8-7.9 (s.4H.ArH). 3.2(s.3H. $\left.\mathrm{CH}_{3}\right) .1 .9-2.4$ (m. 3H. $\mathrm{CH}_{3}$ ).

While reaction of mole of (I) with two moles of biacetyl afforded (VII). Benzoin as an example of aromatic ketone. condensed with (I) to give (VIII) (Schem I).

Maleic anhydride was reported to react easily with $\mathrm{N}$-aminophthalimide (I) Via ring opening (6). this investigation was extended to involve the reaction of $\mathrm{N}$-aminophthalimide (I) with other anhydrides. namely. itaconic and citraconic anhydrides.

The reaction proceeded smoothly givening the acids (IX) and (X) respectively. These acids are the products of preferential attack of (I) on the carbonyl groups of anhydride remote from the methylene and methyl groups. The alternative acidic structures (XI) and (XII) were rejected since in case of itaconic anhydride the two carbonyl groups are attached to a saturated and an unsaturated carbon atom respectively.

The presence of the methylene group in conjugation with the carbonyl groups leads to decrease of the electrophilicity of that carbonyl. In comparison the remot carbonyl is not 
affected. but becomes more succeptible to attack by the reagent.

A similar behaviour in the reaction of itaconic anhydride and amines was previously reported [10].

In case of citraconic anhydride. the $+I$ effect of the methyl group is expected to minimize the electrophilicity of the adjacent carbonyl group leaving the remote carbonyl group more susceptible for attacke (Schem 2).

The acidic character of these products was confirmed by their ready solubility in aqueous sodium carbonate solution and reprecipitation by the addation of ice-cold hydrochloric acid.

$\mathrm{N}$-Arylmethyleneaminophthalimides are known to undergo cleavage under the influence of some Grignard reagent [4] This behaviour tempted us to investigate the reaction of phthalimide derivatives (II) with primary and secondary amines. It was found that these compounds underwent complete cleavage under the influence of $n$-butylamine and secondary amine. namely. piperidine to give $\left(\mathrm{XIII}_{2-c}\right)$. This reaction apparently proceeded by nucleophilic attack on only one carbonyl group (Schem 2).

The reaction of phenyl magnesium bromide with $\mathrm{N}$-arylmethyleneamino phthalimides II and $\left(\mathrm{IV}_{\mathrm{ac}}\right)$ proceeded via refluxing to give $\left(\mathrm{XIV}_{\mathrm{ac}}\right)$.

The other tautomeric open chain structure (XV) was rejected (Schem 1) since such structure would show two carbonyl stretching frequencies and sharp absorption of $\mathrm{NH}$ group. The possibility of 1 . 2-addition to the $-\mathrm{C}=\mathrm{N}$ - group was also excluded because of the absence of carbonyl stretching frequencies of $\mathrm{N}$-substituted phthalimides and the existence of the $-\mathrm{C}=\mathrm{N}$ absorptioins in their infrared spectra (C.F. table 1).

\section{Experimental}

All melting points are not corrected. 'HNMR spectra on a EMNMR spectrometer 200 $\mathrm{MH}_{2}$ PMR using DMSO as a solvent and TMS as internal reference (chemical shifts in $\delta \mathrm{ppm}$ ). I.R. spectra in $\mathrm{KBr}$ were recorded on pye Unicam SP 1200 Spectrophotometer. Analyses were made in Reasarch Micro analytical laboratory of Cairo University.

\section{General Method}

\section{Reaction with carbonyl compounds}

A mixture of (1) $(0.01 \mathrm{~mol})$ and aldehydes or ketones $(0.01 \mathrm{~mol})$ in ethanol $(30 \mathrm{ml})$ was heated under reflux for $2 \mathrm{hrs}$ and left to cool. The crystalline solid formed was seperated. and crystallized from the appropriate solvent to give [II - VIII] and XIII (C.F. Table 1).

\section{Reaction with itaconic and citraconic anhydrides}

A mixture of (1) $(0.01 \mathrm{~mol})$ and anhydrides $(0.01 \mathrm{~mol})$ in benzene $(30 \mathrm{ml})$ was heated under reflux for two hours. and left to cool. The solid obtained was filtered off and crystallised from the appropriate solvent to give IX . XI (C.F. Table 1).

Action of amines on $\mathrm{N}$-arylmethyleneaminophthalimides.

A solution of $\mathrm{N}$-arylmethyleneaminophthalimides $(0.01 \mathrm{~mol})$ in ethanol $(30 \mathrm{ml})$ was treated with the amine $(0.01$ mole) and the reaction mixture was left overnight at room temperature with occasional shaking. The product obtained was crystallized from the appropriate solvent to give XIIIa-c (C.F. Table 1). 
Table 1 : physical deata of compounds

\begin{tabular}{|c|c|c|c|c|c|c|c|c|}
\hline \multirow[t]{2}{*}{$\begin{array}{l}\text { Comp- } \\
\text { ound }\end{array}$} & \multirow[t]{2}{*}{ m.p. $\left(C^{0}\right)$} & \multirow{2}{*}{$\begin{array}{c}\text { Solvent } \\
\text { of } \\
\text { cryst.a }\end{array}$} & \multirow[t]{2}{*}{$\begin{array}{l}\text { Yield } \\
(\%)\end{array}$} & \multirow[t]{2}{*}{$\begin{array}{l}\text { Molecular } \\
\text { Formula }\end{array}$} & \multicolumn{3}{|c|}{$\begin{array}{c}\text { Analysis } \\
\text { Calcd / Found. \% }\end{array}$} & \multirow[t]{2}{*}{ i.r. $\left(\mathrm{cm}^{-1}\right)$} \\
\hline & & & & & $\mathrm{C}$ & $\mathrm{H}$ & $\mathrm{N}$ & \\
\hline \multirow[t]{2}{*}{ II } & 180 & B & 55 & $\mathrm{C}_{12} \mathrm{H}_{10} \mathrm{~N}_{2} \mathrm{O}_{2}$ & 67.28 & 4.67 & 13.08 & $v_{C}=01730.1785$ \\
\hline & & & & & 67.50 & 5.00 & 12.80 & $v_{C}=N 1640$ \\
\hline \multirow[t]{2}{*}{ III } & $228-230$ & $\mathrm{E}$ & 40 & $\mathrm{C}_{12} \mathrm{H}_{12} \mathrm{~N}_{2} \mathrm{O}_{3}$ & 62.06 & 5.17 & 11.05 & $v_{C}=01730.1785$ \\
\hline & & & & & 62.50 & 5.10 & 11.60 & $v_{\mathrm{NH} . \mathrm{OH}} 3225-3320$ \\
\hline \multirow[t]{2}{*}{$\Gamma \mathrm{V}_{\mathrm{a}}$} & 233 & E & 53 & $\mathrm{C}_{18} \mathrm{H}_{11} \mathrm{~N}_{3} \mathrm{O}_{2}$ & 71.76 & 3.65 & 13.9 & $v_{C}=01680-1675$ \\
\hline & & & & & 72.3 & 4.0 & 14.2 & $v_{C}=N 1630$ \\
\hline \multirow[t]{2}{*}{$I_{\mathrm{b}}$} & 167 & E & 49 & $\mathrm{C}_{18} \mathrm{H}_{16} \mathrm{~N}_{2} \mathrm{O}_{5}$ & 63.52 & 4.70 & 8.21 & $v_{C}=01690.1680$ \\
\hline & & & & & 64.38 & 4.6 & 8.26 & $v_{C}=N 1640$ \\
\hline \multirow[t]{2}{*}{$\mathrm{IV}_{\mathrm{c}}$} & 210 & E & 42 & $\mathrm{C}_{18} \mathrm{H}_{16} \mathrm{~N}_{2} \mathrm{O}_{2}$ & 73.97 & 5.47 & 9.58 & $v_{C}=01680.1675$ \\
\hline & 200 & & & & 74.26 & 6.64 & 9.59 & $v_{C}=N 1640$ \\
\hline$I_{\text {d }}$ & 298 & B-E & 47 & $\mathrm{C}_{17} \mathrm{H}_{13} \mathrm{~N}_{2} \mathrm{O}_{3}$ & $\begin{array}{l}66.44 \\
6.10\end{array}$ & 4.23 & 13.68 & $v_{C}=0 \quad 1710.1705 .1690$ \\
\hline \multirow{2}{*}{ V } & 330 & $\mathbf{R}$ & 53 & . & $\begin{array}{l}66.19 \\
6870\end{array}$ & $\begin{array}{l}4.10 \\
3.30\end{array}$ & 15.11 & $v_{C}=N 1675$ \\
\hline & & & & $\left.C_{241} n_{14}\right)_{4} U_{4}$ & 68.23 & 3.53 & & \\
\hline \multirow[t]{2}{*}{ VI } & 165 & $E$ & 70 & $\mathrm{C}_{12} \mathrm{H}_{10} \mathrm{~N}_{2} \mathrm{O}_{3}$ & 62.60 & 4.34 & 12.17 & \\
\hline & & & & & 62.81 & 4.13 & 12.00 & $v_{C}=01758.1782$ \\
\hline \multirow[t]{2}{*}{ VII } & decomp & B & 82 & $\mathrm{C}_{20} \mathrm{H}_{12} \mathrm{~N}_{4} \mathrm{O}_{4}$ & 64.17 & 3.74 & 14.97 & $v_{C}=N 1607$ \\
\hline & at 220 & & & & 63.61 & 3.52 & 14.14 & $v_{C}=N 1607$ \\
\hline \multirow[t]{2}{*}{ VIII } & 188 & B & 79 & $\mathrm{C}_{22} \mathrm{H}_{16} \mathrm{~N}_{2} \mathrm{O}_{3}$ & 74.15 & 4.49 & 7.86 & \\
\hline & & & & & 74.10 & 4.80 & 7.90 & $\begin{array}{l}v_{C}=01670.1735 .1790 \\
v \mathrm{OH}_{3} 3390\end{array}$ \\
\hline \multirow[t]{2}{*}{ IX } & 168 & E & 71 & $\mathrm{C}_{13} \mathrm{H}_{10} \mathrm{~N}_{2} \mathrm{O}_{5}$ & 56.90 & 3.64 & 10.21 & $v_{C}=01700.1740 .1770$ \\
\hline & & & & & 56.30 & 3.80 & 10.50 & $v_{\mathrm{OH}} . \mathrm{NH} 3190,3280$ \\
\hline \multirow[t]{2}{*}{ XI } & 180 & B.E & 42 & $\mathrm{C}_{13} \mathrm{H}_{10} \mathrm{~N}_{2} \mathrm{O}_{3}$ & 56.90 & 3.64 & 10.21 & $v_{C}=01710.1758 .1770$ \\
\hline & & & & & 56.80 & 3.30 & 10.7 & $v_{\mathrm{NH}} . \mathrm{OH} 3120.3230$ \\
\hline \multirow[t]{2}{*}{$\mathrm{XIII}_{\mathbf{a}}$} & $180-181$ & B & 72 & $\mathrm{C}_{16} \mathrm{H}_{21} \mathrm{~N}_{2} \mathrm{O}_{3}$ & 70.23 & 7.69 & 10.25 & $v_{\mathrm{NH}} \quad 3240$ \\
\hline & & & & & 69.26 & 8.44 & 10.13 & $v_{C}=01630$ \\
\hline \multirow[t]{2}{*}{$\mathrm{XIII}_{\mathrm{b}}$} & $216-218$ & $\mathrm{E}$ & 68 & $\mathrm{C}_{17} \mathrm{H}_{23} \mathrm{~N}_{3} \mathrm{O}_{3}$ & 64.35 & 7.25 & 13.2 & $v_{\mathrm{NH}} \quad 3200$ \\
\hline & & & & & 63.57 & 6.67 & 12.86 & $v_{C}=01660$ \\
\hline \multirow[t]{2}{*}{$\mathrm{XIII}_{\mathrm{c}}$} & $198-199$ & $\mathrm{E}$ & 55 & $\mathrm{C}_{23} \mathrm{H}_{27} \mathrm{~N}_{5} \mathrm{O}_{3}$ & 64.96 & 6.35 & 9.88 & $v_{\mathrm{NH}} \quad 3200$ \\
\hline & & & & & 65.21 & 6.27 & 9.20 & $v_{C}=01670$ \\
\hline \multirow[t]{2}{*}{$\mathrm{XIV}_{\mathrm{a}}$} & $240-241$ & B-LP & 36 & $\mathrm{C}_{18} \mathrm{H}_{16} \mathrm{~N}_{2} \mathrm{O}_{2}$ & 73.72 & 5.46 & 9.55 & $v_{\mathrm{NH}} \quad 3200$ \\
\hline & $270-271$ & $T^{-I} P$ & 13 & $\mathrm{C}_{-} \mathrm{H}_{-} \mathrm{N}_{2} \mathrm{O}_{2}$ & 74.12 & 5.59 & 9.56 & $v_{C}=01675$ \\
\hline $\mathrm{XIV}_{\mathrm{b}}$ & $210-211$ & $1-L P$ & 43 & $\mathrm{C}_{24} \mathrm{H}_{17} \mathrm{~N}_{2} \mathrm{O}_{3}$ & \begin{tabular}{|l|}
15.98 \\
70.34
\end{tabular} & $\begin{array}{l}4.48 \\
4.15\end{array}$ & $\begin{array}{l}11.08 \\
1169\end{array}$ & $v_{\mathrm{NH}} \quad 3290$ \\
\hline \multirow[t]{2}{*}{$\mathrm{XIV}_{\mathrm{c}}$} & $764-266$ & B-LP & 51 & $\mathrm{C}_{24} \mathrm{H}_{22} \mathrm{~N}_{2} \mathrm{O}_{2}$ & 68.89 & 5.26 & 6.69 & $v_{C}=01680$ \\
\hline & & & & & 68.45 & 5.29 & 6.46 & $v_{\mathrm{NH}} \quad 3320$ \\
\hline
\end{tabular}

$\mathrm{B}=$ Benzene $. \mathrm{E}=$ Ethanol $. \mathrm{B}-\mathrm{LP}=$ Benzene - Light pet. (b.p. 80-100 $\mathrm{C}^{\circ}$ ).

$\mathrm{T}$-LP $=$ Toluene - Light pet. (b.p. $100-120 \mathrm{C}^{\circ}$ ). 


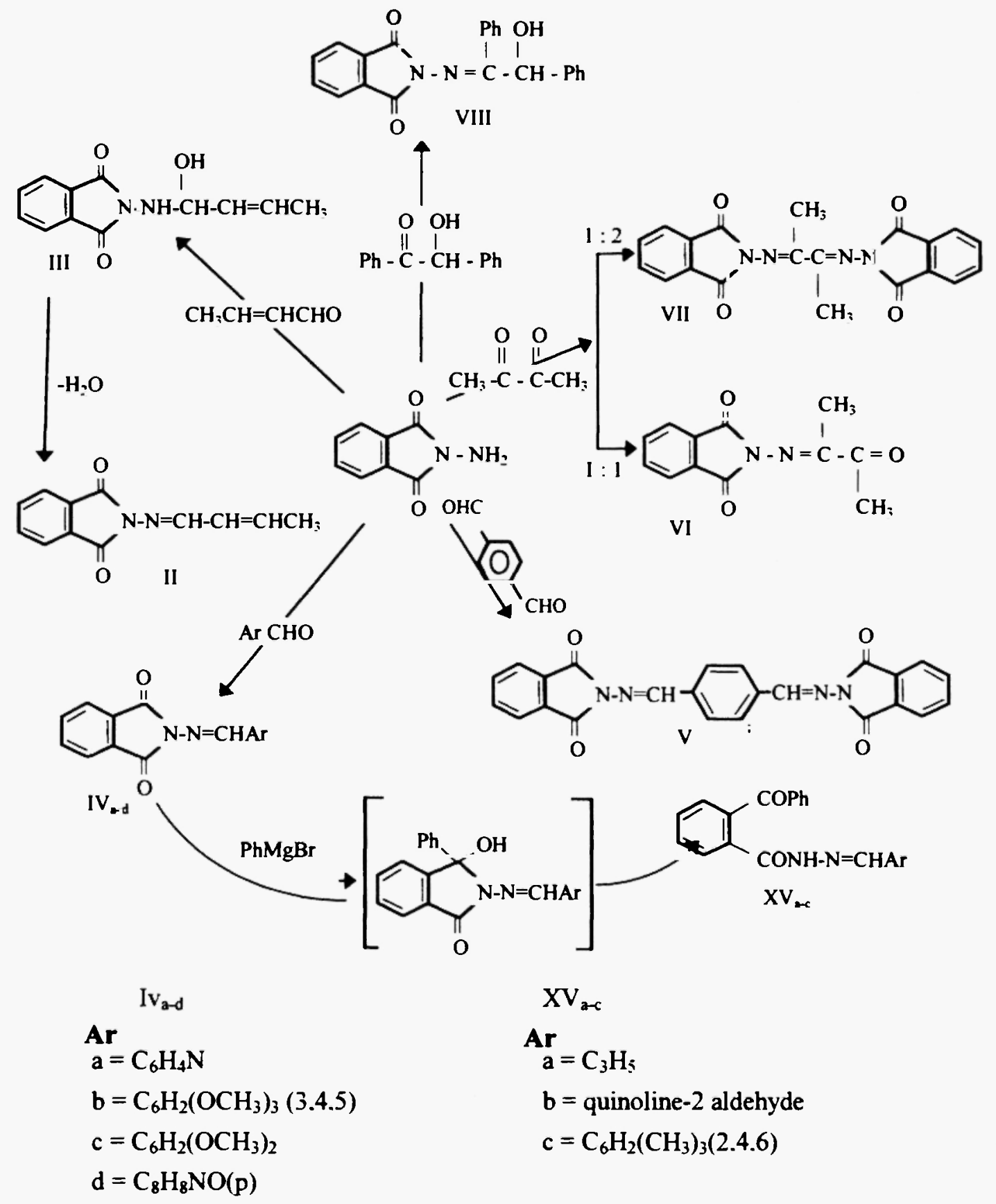

(Scheme 1) 


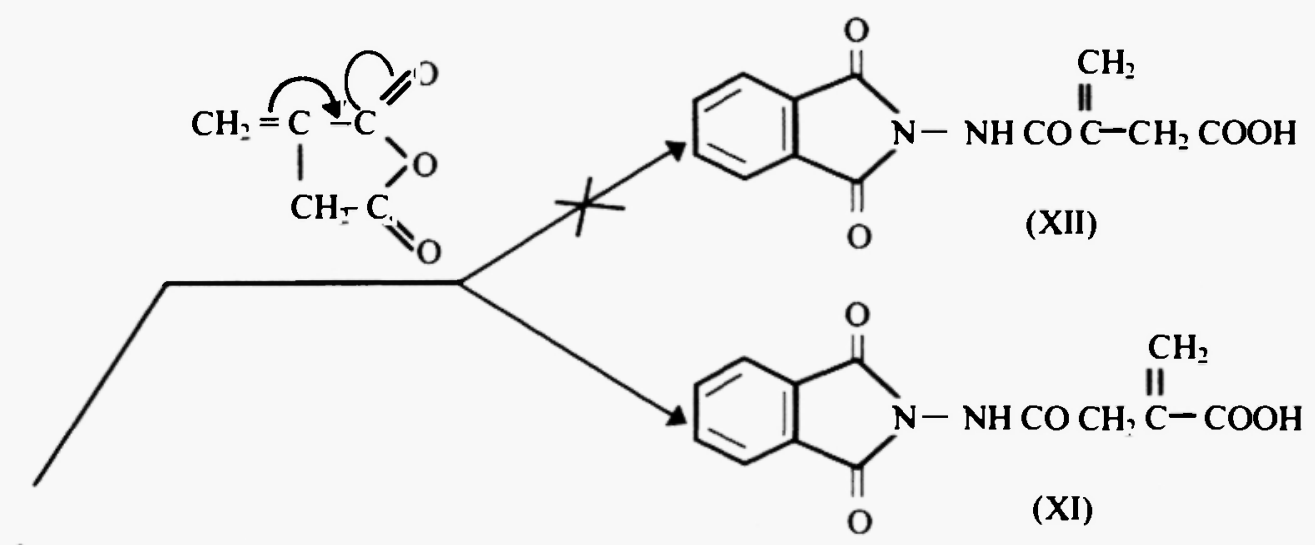

1
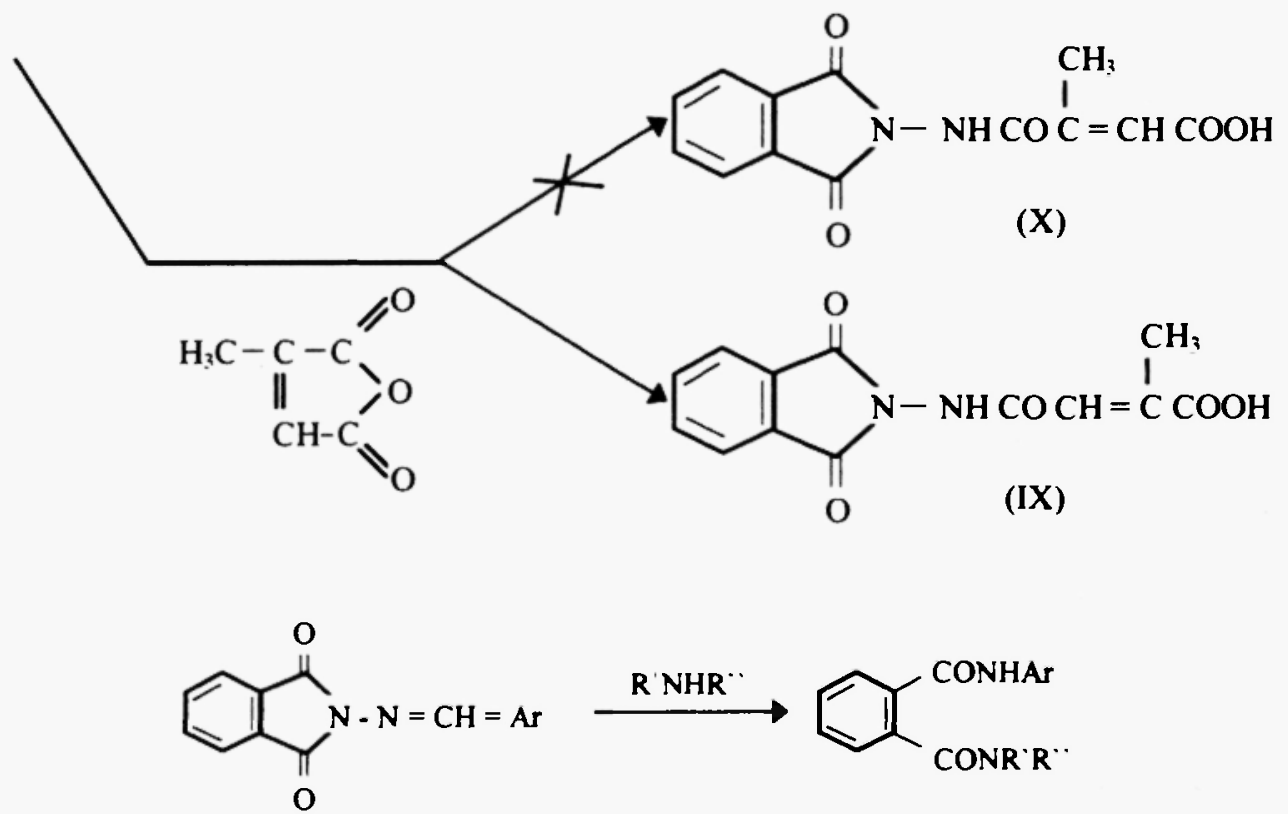

$\begin{array}{rlrl} & \text { Ar } & R^{\prime} & \mathbf{R}^{\prime} \\ \quad \mathbf{a}=\mathrm{C}_{3} \mathrm{H}_{5} & \mathrm{H} & \mathrm{C}_{4} \mathrm{H}_{9} \\ \mathbf{b}=\mathrm{C}_{3} \mathrm{H}_{7} \mathrm{O} & - & \mathrm{C}_{5} \mathrm{H}_{10} \\ c=\mathrm{C}_{9} \mathrm{H}_{11} \mathrm{O}_{3} & - & \mathrm{C}_{5} \mathrm{H}_{10}\end{array}$

(Scheme 2) 


\section{Action of Grignard reagents on $\mathbf{N}$-(arylmethyleneamino) phthalimides}

A solution of phenylmagnesium bromide $(0.03 \mathrm{~mol})$ in dry ether $(30 \mathrm{ml})$ was added to a solution of $\mathrm{N}$-arylmethyleneaminophthalimieds $(0.01 \mathrm{~mol})$ in dry benzene $(50 \mathrm{ml})$ with occasional shaking. The reaction mixture was heated under reflux on a boiling water bath for $5 \mathrm{hrs}$. and left overnight. The reaction mixture was then hydrolysed by shaking with a saturated solution of ammonium chloride. The benzene - ether layer was washed several times with cold water. seperated. dried over anhydrous sodium sulphate. filtrate. and concentrated under reduced pressure and left to cool. The solid formed was filtered off and crystallised from suitable solvent to give XVa-c (C.F. Table 1).

\section{References}

(1) E.. Profft. Teubner. H. and Weuffen. H.. Arch. Exp. Velerinaermed.. 21.225 (1967).

(2) O. T.. Suvulescu. Barbu. V. and Tudoescu. phytopathol. Z.. 59. 129. (1967).

(3) J. Porszaz. and Nador. K.. Arzeneimitted - Forsch.. 6. 695 (1958).

(4) R.F.. Collins. Davis. M.. Edge. N.D. Hill. J.. Reading. H.W. and Turn bull, E.R.. Brit . J. Phamacol.. 14. 467 (1956).

(5) W.I.. Awad. Ismail. M.F.. and Kandile. N.G.. Aust. J. chem. 281621 (1975).

(6) M.F.. Ismail. and Kandile. N.G.. J. Ind. Chem. 21 B 46 (1982).

(7) N.G.. Kandile. Acta. Chim. Hung. 126(4). 533 (1989).

(8) N.G.. Kandile. and Wassef. Annual Review. Ain Shams Uni.. Uni. college for Girls. Science Section. 16. 85 (1991).

(9) V. J.. Rothenburg. Prakt. Chem. 51 . 151 (1895).

(10) A.. Zilkha. and Golik. V.. J. Org. Chem.. 28 : 2007 (1963).

Received on February 20, 2002 